\title{
PRODUÇÃO E SAZONALIDADE DE PESSEGUEIRO E NECTARINEIRA SOB FLORESCIMENTO ESPONTÂNEO E COM CIANAMIDA HIDROGENADA E ÓLEO MINERAL ${ }^{1}$
}

\author{
SARITA LEONEL ${ }^{2} \&$ MARCO ANTONIO TECCHIO 3
}

RESUMO - O trabalho avaliou a produção e a sazonalidade de cultivares de pessegueiro e nectarineira, com e sem o uso de cianamida hidrogenada, em dois ciclos de produção (2009 e 2010). O experimento foi desenvolvido na Faculdade de Ciências Agronômicas, UNESP, Câmpus de Botucatu-SP, localizado nas seguintes coordenadas geográficas: latitude $22^{\circ} 51^{\prime} 55^{\prime}$ 'S, longitude $48^{\circ} 26^{\prime} 22^{\prime \prime} \mathrm{O}$ e $810 \mathrm{~m}$ de altitude. O tipo climático predominante é o temperado quente (mesotérmico), com chuvas no verão e seca no inverno. A aplicação de cianamida hidrogenada e óleo mineral resultou numa antecipação na data do início da colheita para todas as cultivares. Também houve uma concentração no período produtivo, diminuição no ciclo de produção e aumento na produção. A avaliação do ciclo da poda à colheita sob florescimento espontâneo evidenciou que as cultivares mais precoces foram: Precocinho (81,5 dias) e Conserva 693 (87 dias). As mais tardias foram: Turmalina (141,5 dias) e CP 951 C (134,5 dias). Com o uso da cianamida hidrogenada e do óleo mineral, o ciclo da poda à colheita permitiu a caracterização das cultivares mais precoces: Precocinho $(87,5$ dias $) \mathrm{e}$ a nectarineira Sun Blaze (95,5 dias). As mais tardios foram: Diamante Mejorado (126,5 dias) e CP 951 C (120 dias). As cultivares Turmalina (20,2 $\left.\mathrm{kg} \mathrm{planta}^{-1}\right)$, Conserva 693 (20,75 kg planta-1) e Aurora 1 (15,65 $\mathrm{kg}$ planta $^{-1}$ ) foram as mais produtivas .

Termos para indexação: Prunus persica var. nucipersica; Prunus persica var. vulgaris, épocas, clima.

\section{YIELD AND HARVEST PERIOD OF PEACH AND NECTARINE CULTIVARS AT FREE BLOOMING AND WITH THE USE OF HYDROGEN CYANAMIDE}

\begin{abstract}
The work evaluated the yield, fruiting time and harvest period of the peach and nectarine cultivars, with and without hydrogen cyanamide, in two crop cycles (2009 and 2010). The experiment was carried out in the School of Agronomical Sciences (FCA), São Paulo State University (UNESP), Botucatu Campus, SP, Brazil, located at $22^{\circ} 51^{\prime} 55^{\prime} \mathrm{S}, 48^{\circ} 27^{\prime} 22^{\prime \prime} \mathrm{W}$ and $810 \mathrm{~m}$ altitude. The predominant climate in the study region is described as a warm temperate climate (mesothermal), with rainy summer and dry winter. The spraying with hydrogen cyanamide and mineral oil showed in the earlier harvest dates for all cultivars evaluated. Also, there was one concentration on the yield period, decrease on the crop cycle and increase the yield productivity. The evaluation of the pruning until harvest time with free blooming showed that the earliest cultivars were: Precocinho (81.5 days) and Conserva 693 (87 days). The latest were: Turmalina (141.5 days) and CP $951 \mathrm{C}$ (120 days). With the spraying with hydrogen cyanamide and mineral oil the pruning until harvest time showed the earliest cultivars: Precocinho ( 87.5 days) and Sun Blaze nectarine ( 95.5 days). The latest were: Diamante Mejorado (126.5 days) and CP $951 \mathrm{C}$ (120 days). The highest yield were observed in the Turmalina (20.2 kg plant-1), Conserva 693 (20.75 kg plant-1) and Aurora 1 (15.65 kg plant $\left.{ }^{-1}\right)$ cultivars. Index terms: Prunus persica var. nucipersica; Prunus persica var. vulgaris, épocas, clima.
\end{abstract}

\footnotetext{
${ }^{1}$ Palestra Sinfruit 077 - Simpósio Internacional de Fruticultura - Avanços na Fruticultura (17 a 21 Outubro) - Trabalho financiado pela FAPESP. Processo: 09-50862-3.

${ }^{2}$ UNESP. Faculdade de Ciências Agronômicas. Departamento de Produção Vegetal. Cx Postal 237. CEP 18610-307. Botucatu-SP. sarinel@fca.unesp.br

${ }^{3}$ Centro Avançado de Pesquisa Tecnológica do Agronegócio de Frutas - Instituto Agronômico de Campinas - CEP 13214-820 - Jundiaí-SP. tecchio@iac.sp.gov.br.
} 


\section{INTRODUÇ̃̃O}

O avanço na fronteira de produção do pessegueiro, para regiões de clima subtropical e de inverno ameno, deve-se principalmente ao lançamento de novas cultivares, menos exigentes em frio hibernal e à criação de novas tecnologias que possibilitem o desenvolvimento da cultura. De acordo com Marodin et al. (2008), o pessegueiro é uma planta de clima temperado que necessita de horas de frio abaixo de $7,2^{\circ} \mathrm{C}$ para que ocorram brotação e florescimento uniformes, sendo que essa quantidade de horas varia conforme a cultivar. O pessegueiro, quando cultivado em regiões com deficiência de frio hibernal, pode apresentar sintomas erráticos, como atraso e maior duração da floração, menor percentual de floração e brotação e, consequentemente, redução na produção, com frutos desuniformes e de baixa qualidade, característica de plantas mal adaptadas (CITADIN et al., 2006).

As cultivares de pessegueiros podem ser classificadas em ultraprecoces, bem precoces, precoces, medianas, tardias e bem tardias, de acordo com a duração do ciclo de desenvolvimento dos frutos e a época em que são colhidos. Devido aos trabalhos de melhoramento genético, aliados às novas tecnologias de cultivo, é possível realizar a colheita de pêssegos de agosto a fevereiro (BARBOSA et al., 2003). O conhecimento do ciclo de desenvolvimento das cultivares é importante para definir práticas culturais, como quebra de dormência, raleio, podas, adubação, tratamento fitossanitário e irrigação, em diferentes regiões, e com isso identificar as cultivares mais bem adaptadas.

Compostos químicos, como óleo mineral, dinitro-ortocresol, tiouréia, nitrato de potássio, nitrato de cálcio, ácido giberélico e citocininas, são citados como efetivos na quebra de dormência de muitas frutíferas, podendo substituir parcialmente a necessidade de frio e estimular a abertura precoce e mais uniforme das gemas. A maior eficiência da cianamida cálcica $\left(\mathrm{CaCN}_{2}\right)$ e da cianamida hidrogenada $\left(\mathrm{H}_{2} \mathrm{CN}_{2}\right)$ sobre os outros compostos advém da presença do radical $-\mathrm{C} \equiv \mathrm{N}$, que é muito mais reativo (PIRES; MARTINS, 2003). Existe necessidade de mais estudos sobre os efeitos desses compostos na cultura do pessegueiro, a depender da região onde está estabelecido o cultivo.

Considerando o potencial econômico do cultivo no Estado de São Paulo, que apresenta como vantagem a colheita antecipada dos frutos, quando comparada com as principais regiões produtoras brasileiras e também com países como Chile, Argentina, Uruguai e África do Sul, adicionada à possibilidade de diversificação de cultivares mais adaptadas ao clima subtropical, cuja adoção poderia possibilitar um aumento na renda e na sazonalidade da oferta do produto, o trabalho teve como objetivos caracterizar o ciclo e quantificar a produção de cultivares de pessegueiro e nectarineira, com e sem o emprego de produto para a quebra da dormência, em região de clima subtropical, no município de Botucatu-SP.

\section{MATERIAL E MÉTODOS}

O trabalho foi realizado na Fazenda Experimental Lageado, da Faculdade de Ciências Agronômicas da Universidade Estadual Paulista - UNESP, situada a $22^{\circ} 51^{\prime} 55^{\prime \prime}$ S., $48^{\circ} 26^{\prime} 22^{\prime \prime}$ O. e a $810 \mathrm{~m}$ de altitude. O clima é do tipo mesotérmico, Cwa, subtropical úmido. A precipitação pluvial média é de $1.433 \mathrm{~mm}$. A umidade relativa do ar é de $71 \%$, com temperatura média anual de $19,3{ }^{\circ} \mathrm{C}$ (CUNHA, 1999). O solo da área é classificado como Nitossolo Vermelho, segundo os critérios da EMBRAPA (1999).

O delineamento experimental foi o de parcelas subdivididas, com 3 repetições em blocos ao acaso, onde as parcelas foram constituídas pelos tratamentos com e sem a aplicação de cianamida hidrogenada e do óleo mineral, e as subparcelas, pelas cultivares de pessegueiro e nectarineira. Foram utilizadas 4 plantas por parcela, as quais estavam rodeadas por plantas na bordadura. Avaliaram-se as cultivares de pessegueiro Aurora 1, Tropic Beauty, Douradão, Dourado 2, Marli e a nectarineira 'Sun Blaze', tradicionalmente cultivadas em São Paulo, as cultivares Turmalina e Precocinho cultivadas no Rio Grande do Sul e algumas possibilidades de diversificação, como as seleções de Cascata (953; 797 e 587) e Conserva 693, provenientes do Rio Grande do Sul, e Diamante Mejorado, CP 9553 CYN e CP 951 C, provenientes do México.

Realizou-se o experimento durante os ciclos agrícolas de 2009 e 2010 . As plantas estavam com 5 anos de idade, cultivadas no espaçamento de $4,0 \mathrm{~m}$ entre linhas e 4,0 m entre plantas. Durante a condução do experimento, foram realizadas práticas culturais e fitossanitárias, seguindo as recomendações técnicas da cultura. Realizou-se a poda de frutificação na primeira semana do mês de julho (2009 e 2010), sendo o raleio realizado duas semanas após a plena floração. Em duas plantas por repetição de cada cultivar, foi utilizado o produto comercial Dormex (cianamida hidrogenada), na dosagem de $0,6 \%$ e $1 \%$ de óleo mineral. A solução foi aplicada na segunda semana de julho (2009 e 2010), no estádio de gemas dormentes, através de pulverização, com o auxílio de uma cortina plástica, para que não houvesse deriva do produto. 
Realizou-se a colheita quando os frutos atingiram o ponto de maturação fisiológica, que correspondeu ao final do mês de setembro até meados de dezembro.

Foram avaliadas as datas da poda até o final da colheita, do início e final das colheitas e o intervalo de colheitas, com a finalidade de caracterização do período produtivo. Além disso, foi mensurada a produção, determinada através do produto do número médio de frutos por planta, e respectivo peso médio (g). Os resultados foram submetidos à análise de variância e, quando houve significância, as médias foram comparadas pelo teste de Tuckey, ao nível de $5 \%$ de probabilidade.

\section{RESULTADOS E DISCUSSÃO}

Os resultados demonstraram que a dosagem de $0,6 \%$ de cianamida hidrogenada e $1 \%$ de óleo mineral não atendeu às necessidades de todas as cultivares para a quebra da dormência. As seleções de Cascata não responderam bem às concentrações dos produtos, talvez exigindo uma dosagem maior, uma vez que as necessidades de frio não foram completamente satisfeitas. Considerando-se o ciclo de produção da poda à colheita (julho a dezembro) no ano de 2009, verificou-se que as médias das temperaturas mínimas, máximas e médias foram: 16,$6 ; 25,5$ e $21,0^{\circ} \mathrm{C}$, respectivamente. No mesmo ciclo avaliado no ano de 2010, as médias foram: 14,6; 27,2 e 21, $1^{\circ} \mathrm{C}$, respectivamente. Verificou-se que, a partir de maio, as temperaturas médias começaram a diminuir. Em junho, foram registradas as menores temperaturas e, em julho, as temperaturas começaram a aumentar novamente, sendo este aumento da temperatura, após um período de baixas temperaturas, juntamente com a aplicação da cianamida hidrogenada, um estímulo para a brotação e o florescimento das cultivares. De acordo com Barbosa et al. (2003), as cultivares adaptadas para as regiões subtropicais não têm necessidade de muitas horas de frio e brotam facilmente, enquanto as cultivares mais exigentes em frio dificilmente brotam em regiões de inverno ameno, como no Estado de São Paulo, pois não conseguem acumular horas de frio suficientes para superar a dormência.

As aplicações de cianamida hidrogenada e de óleo mineral resultaram na antecipação da data do início da colheita para todas as cultivares. Também houve uma concentração no período produtivo e diminuição no ciclo de produção para todas as cultivares, com exceção de Marli e Dourado-2, nas quais o ciclo foi o mesmo, independentemente do uso dos produtos, no ciclo 2009 (Tabela 1). Em 2010, os efeitos mais pronunciados foram observados na cultivar Turmalina, na qual houve redução no ciclo de produção de 57 dias com o uso da cianamida hidrogenada e do óleo mineral (Tabela 2). Os efeitos benéficos do emprego da cianamida hidrogenada e do óleo mineral já foram relatados por diversos autores, a depender da dosagem empregada, das necessidades em horas de frio e da época de aplicação. De maneira geral, quanto maior as necessidades em frio das cultivares que não são oferecidas pelos locais de cultivo, maiores devem ser as dosagens dos produtos, evidenciando a necessidade da pesquisa local (NAVA et al., 2009).

Em trabalho com a cultivar de pessegueiro Chiripá, em Pato Branco-PR, Citadin et al. (2006) concluíram que os tratamentos que receberam $\mathrm{CH} 2 \mathrm{~N} 2$, anteciparam o pico de colheita em uma semana, comparado àqueles que não receberam, confirmando os resultados obtidos neste trabalho, no qual se verificou que a plena brotação das gemas vegetativas ocorreu simultaneamente ao pleno florescimento da maioria das cultivares. As cultivares com florescimento espontâneo, mais precoces, foram: Cascata 953, que apresentou um ciclo da poda à colheita de 86 dias, e Precocinho com 92 dias. Com o uso da cianamida hidrogenada, os resultados foram praticamente os mesmos: 83 e 84 dias, respectivamente. Quando se avaliaram as cultivares mais tardias, observou-se que foram: Turmalina e CP 951 $\mathrm{C}$, que apresentaram um ciclo de 147 e 141 dias, com florescimento espontâneo, respectivamente. Com o emprego da cianamida hidrogenada, os resultados demonstraram que alguns genótipos apresentaram maior eficiência ao uso do produto. Dessa maneira, a cultivar de pessegueiro mais tardio foi Diamante Mejorado, com ciclo da poda à colheita de 129 dias (Tabela 3).

De acordo com Marodin et al. (2008), 'Diamante Mejorado' apresentou um ciclo de 100 dias entre a floração e a colheita, na depressão central do Rio Grande do Sul, com pleno florescimento em 03-08 e concentração da colheita em 04-11, sendo que tais resultados são concordantes com os obtidos neste trabalho, em que o pleno florescimento ocorreu no início de agosto, e o ciclo do florescimento à colheita foi de 103 dias, aproximadamente. Segundo os mesmos autores, as cultivares de origem mexicana, como é o caso do Diamante Mejorado, CP 9553 CYN e CP 951-C, destacam-se pela boa adaptação ao clima subtropical.

Avaliando o comportamento do pessegueiro 'Douradão' em Itupeva-SP, Barbosa et al. (1999) reportaram que as plantas floresceram entre $1^{\circ}$ e 10 de julho, e os frutos amadureceram no segundo decênio de outubro. Os resultados obtidos nesta pesquisa corroboram os dados obtidos pelos autores, uma vez 
que o início da colheita, sob florescimento espontâneo, deu-se em 20-10-2011. Da mesma maneira, os dados do presente experimento assemelham-se nos encontrados por Pedro Júnior et al. (2007), os quais relatam que a época de florescimento espontâneo dos principais pessegueiros da persicultura paulista, como 'Aurora-1', 'Douradão' e 'Dourado-1', ocorre entre a segunda e terceira semana de julho.

Quando foi efetuada a avaliação conjunta do intervalo de colheita das cultivares nos dois ciclos de avaliação, verificou-se que, em média, o mesmo foi de 23,4 dias sob florescimento espontâneo e de 19,5 dias com a utilização da cianamida hidrogenada e óleo mineral. O menor intervalo de colheita sem o uso da cianamida hidrogenada foi observado na nectarineira 'Sun Blaze' (14 dias) e no pessegueiro CP 9553 CYN (16,5 dias). O maior intervalo foi observado na seleção de pessegueiro Cascata 797 (44 dias), sendo que essa foi uma característica importante para inferir sobre a não adaptação dessa seleção à região de Botucatu-SP, uma vez que a colheita foi bastante desuniforme, num longo período e com baixa produção. Com a aplicação dos produtos, o menor intervalo de colheita foi observado em Cascata 587 (10 dias) e em 'Sun Blaze' (12,5 dias). O maior intervalo foi verificado, também, na seleção de pessegueiro Cascata 797 (30,5 dias).

Os genótipos avaliados apresentaram, em média, um ciclo de 119 dias da poda à colheita, quando foi avaliado o florescimento espontâneo. Em média, com o emprego da cianamida hidrogenada, as cultivares apresentaram um ciclo de 106 dias, evidenciando resultados de adiantamento do ciclo de produção de 13 dias. Na avaliação conjunta dos dois ciclos de produção, houve uma redução média do ciclo da poda à colheita de 10,5 dias para todas as cultivares. Conforme o relato de Almeida e Durigan (2006), 62,35\% dos pêssegos comercializados no Entreposto Terminal de São Paulo, da CEAGESP, são oriundos de colheitas efetuadas entre a segunda quinzena de outubro e a primeira quinzena de novembro. Os autores salientaram ainda que, embora a safra paulista tenha duração de até 16 semanas, o maior volume é proveniente das quatro semanas reportadas. A grande maioria dos materiais avaliados no trabalho foram colhidos também nesse período, sendo que a avaliação do intervalo entre o início e o final das colheitas, caracterizada pelo período produtivo, mostrou um intervalo de 26,5 dias, em média, para todas as cultivares, sob florescimento espontâneo, e de 23,7 dias com o emprego da cianamida hidrogenada no ciclo de 2010 (Tabela 2).

A produção do pessegueiro pode variar em função dos tratos culturais utilizados, das cultivares e do grau de adaptação destas em função da região de cultivo. De acordo com Marodin et al. (2008), para as cultivares de pessegueiro, a média de produção acima de $10 \mathrm{~kg}$ por planta pode ser considerada alta; de 5 a $10 \mathrm{~kg}$ por planta, a produção é considerada boa, e inferior a $5 \mathrm{~kg}$ por planta, pode ser considerada média. Baseado nesse critério, a maioria das cultivares avaliadas, em média, apresentaram boa produção (12 $\mathrm{kg}_{\text {planta }}{ }^{-1}$ ) (Tabela 3). Contudo, houve diferenças entre cultivares e seleções, nas quais Turmalina, Cascata 587, Conserva 693, Aurora 1, Douradão, Dourado 2, Sun Blaze e CP 951-C apresentaram altas produções, acima de $10 \mathrm{~kg}$ por planta, Cascata 953, cascata 797, Precocinho, Diamante Mejorado, CP 9553-CYN e Marli apresentaram boas produções, situadas entre 5-10 kg por planta.

Em Vista Alegre do Alto (SP), Pereira e Mayer (2008) relataram produções de 22,0 (2005) e 11,35 kg por planta (2006) para 'Aurora 1'. Segundo os autores, a queda de produção de um ano para o outro, ocorreu devido às altas temperaturas, que prejudicaram a frutificação, uma vez que, nos dois anos de avaliação, foi empregada somente uma dose de cianamida hidrogenada. No presente experimento, Aurora 1 produziu, em média, nos dois ciclos de avaliação, $19,6 \mathrm{~kg}$ por planta com o emprego da cianamida hidrogenada e $11,7 \mathrm{~kg}$ por planta sob florescimento espontâneo. Esses resultados implicam o aumento de $40 \%$ em produção com o uso da cianamida hidrogenada e do óleo mineral.

A produção obtida variou em função da aplicação de cianamida hidrogenada e óleo mineral, sendo superior quando do uso dos produtos (Tabela $3)$. Os resultados obtidos para algumas cultivares são inferiores às médias dos produtores de pêssego e nectarina; mas, para outros, as produções podem ser consideradas satisfatórias, indicando a possibilidade da diversificação das cultivares. 
TABELA 1 - Datas do início e final das colheitas, intervalo de colheitas e ciclo da poda ao final das colheitas de cultivares de pessegueiro e nectarineira, com ou sem a aplicação de cianamida hidrogenada. FCA/UNESP/Botucatu. 2009.

\begin{tabular}{|c|c|c|c|c|c|c|}
\hline Cultivar & $\begin{array}{l}\text { Datas do início } \\
\text { e final das } \\
\text { colheitas }\end{array}$ & $\begin{array}{l}\text { Intervalo } \\
\text { de } \\
\text { colheita } \\
\text { (dias) }\end{array}$ & $\begin{array}{l}\text { Ciclo da } \\
\text { poda ao } \\
\text { final da } \\
\text { colheita } \\
\text { (dias) }\end{array}$ & $\begin{array}{c}\text { Datas do início } \\
\text { e final das } \\
\text { colheitas }\end{array}$ & $\begin{array}{l}\text { Intervalo } \\
\text { de } \\
\text { colheita } \\
\text { (dias) }\end{array}$ & $\begin{array}{l}\text { Ciclo da } \\
\text { poda ao } \\
\text { final da } \\
\text { colheita } \\
\text { (dias) }\end{array}$ \\
\hline & \multicolumn{3}{|c|}{ Sem Cianamida Hidrogenada } & \multicolumn{3}{|c|}{ Com Cianamida Hidrogenada } \\
\hline Turmalina & $29-10$ a $28-11$ & 30 & 136 & $13-10$ a $03-11$ & 21 & 111 \\
\hline Cascata 953 & $06-10$ a $25-10$ & 19 & 102 & $1^{\circ}-10$ a $14-10$ & 13 & 91 \\
\hline Cascata 587 & $19-10$ a $05-11$ & 17 & 113 & $12-10$ a $20-10$ & 8 & 97 \\
\hline Cascata 797 & $06-10$ a $15-11$ & 40 & 123 & $1^{\circ}-10$ a $30-10$ & 30 & 107 \\
\hline Conserva 693 & $19-09$ a $25-10$ & 36 & 102 & $12-09$ a $10-10$ & 28 & 87 \\
\hline Precocinho & $18-09$ a $06-10$ & 18 & 83 & $14-09$ a $06-10$ & 15 & 79 \\
\hline Diamante Mejorado & $04-11$ a $20-11$ & 16 & 128 & $30-10$ a $16-11$ & 17 & 124 \\
\hline Aurora-1 & $20-10$ a $07-11$ & 19 & 115 & $14-10$ a $30-10$ & 16 & 107 \\
\hline CP 9553 CYN & 06-10 a $20-10$ & 14 & 97 & $1^{\circ}-10$ a $14-10$ & 13 & 91 \\
\hline CP $951 \mathrm{C}$ & $05-11$ a $20-11$ & 15 & 128 & $30-10$ a $15-11$ & 16 & 123 \\
\hline Tropic Beauty & $15-10$ a $30-10$ & 15 & 107 & $02-10$ a $15-10$ & 13 & 92 \\
\hline Douradão & $26-10$ a $07-11$ & 12 & 115 & $30-10$ a $04-11$ & 04 & 112 \\
\hline Dourado -2 & $15-10$ a $04-11$ & 20 & 112 & $21-10$ a $04-11$ & 13 & 112 \\
\hline Marli & $05-10$ a $28-10$ & 23 & 105 & $14-10$ a $28-10$ & 14 & 105 \\
\hline Sun Blaze (nectarina) & $12-10$ a $22-10$ & 10 & 99 & $16-10$ a $26-10$ & 10 & 95 \\
\hline
\end{tabular}


TABELA 2 - Datas do início e final das colheitas, intervalo de colheitas e ciclo da poda ao final das colheitas de cultivares de pessegueiro e nectarineira, com ou sem a aplicação de cianamida hidrogenada. FCA/UNESP/Botucatu. 2010.

\begin{tabular}{|c|c|c|c|c|c|c|}
\hline Cultivar & $\begin{array}{c}\text { Datas do } \\
\text { início e final } \\
\text { das colheitas }\end{array}$ & $\begin{array}{c}\text { Intervalo } \\
\text { de } \\
\text { colheita } \\
\text { (dias) }\end{array}$ & $\begin{array}{l}\text { Ciclo da } \\
\text { poda ao } \\
\text { final da } \\
\text { colheita } \\
\text { (dias) }\end{array}$ & $\begin{array}{c}\text { Datas do início } \\
\text { e final das } \\
\text { colheitas }\end{array}$ & $\begin{array}{l}\text { Intervalo } \\
\text { de } \\
\text { colheita } \\
\text { (dias) }\end{array}$ & $\begin{array}{c}\text { Ciclo da } \\
\text { poda ao } \\
\text { final da } \\
\text { colheita } \\
\text { (dias) }\end{array}$ \\
\hline & \multicolumn{3}{|c|}{ Sem Cianamida Hidrogenada } & \multicolumn{3}{|c|}{ Com Cianamida Hidrogenada } \\
\hline Turmalina & $25-10$ a $05-12$ & 41 & 147 & $07-10$ a $08-11$ & 32 & 90 \\
\hline Cascata 953 & $03-10$ a $23-10$ & 20 & 86 & $29-09$ a $15-10$ & 16 & 83 \\
\hline Cascata 587 & $17-10$ a $08-11$ & 22 & 123 & $10-10$ a $22-10$ & 12 & 106 \\
\hline Cascata 797 & $02-10$ a $19-11$ & 48 & 134 & $25-09$ a $20-10$ & 31 & 104 \\
\hline Conserva 693 & $08-09$ a $20-10$ & 42 & 104 & 05-09 a 03-10 & 28 & 87 \\
\hline Precocinho & $12-09$ a $08-10$ & 26 & 92 & 05-09 a 30-09 & 25 & 84 \\
\hline Diamante Mejorado & $06-11$ a $25-11$ & 19 & 140 & $1^{\circ}-11$ a $14-11$ & 13 & 129 \\
\hline Aurora-1 & $23-10$ a $19-11$ & 27 & 134 & $10-10$ a $06-11$ & 26 & 121 \\
\hline CP 9553 CYN & $10-10$ a $29-10$ & 19 & 113 & $25 / 09$ a $18-10$ & 23 & 102 \\
\hline CP $951 \mathrm{C}$ & $30-10$ a $26-11$ & 27 & 141 & $26-09$ a $02-11$ & 37 & 117 \\
\hline Tropic Beauty & $05-10$ a $30-10$ & 25 & 114 & $25-09$ a $25-10$ & 30 & 109 \\
\hline Douradão & $20-10$ a $14-11$ & 25 & 129 & $10-10$ a $07-11$ & 28 & 122 \\
\hline Dourado -2 & $10-10$ a $1^{\circ}-11$ & 22 & 116 & $06-10$ a $29-10$ & 23 & 107 \\
\hline Marli & $08-10$ a $25-10$ & 17 & 109 & $30-09$ a $15-10$ & 16 & 99 \\
\hline Sun Blaze (nectarina) & $05-10$ a $23-10$ & 18 & 107 & $27-09$ a $12-10$ & 15 & 96 \\
\hline
\end{tabular}


TABELA 3 - Resultados médios da produção $\left(\mathrm{kg} \mathrm{planta}^{-1}\right)$ de pessegueiro e nectarineira com ou sem a aplicação de cianamida hidrogenada. FCA/UNESP/Botucatu. 2009 e 2010.

\begin{tabular}{|c|c|c|c|c|c|c|}
\hline & \multicolumn{3}{|c|}{$\begin{array}{l}\left.\text { Produção (kg planta }{ }^{-1}\right) \\
2009 \\
\end{array}$} & \multicolumn{3}{|c|}{$\begin{array}{c}\text { Produção (kg/planta) } \\
2010\end{array}$} \\
\hline & \multicolumn{3}{|c|}{ Cianamida Hidrogenada } & \multicolumn{3}{|c|}{ Cianamida Hidrogenada } \\
\hline & Com & Sem & Média & Com & Sem & Média \\
\hline Turmalina & $28,5 \mathrm{Aa}$ & $13,8 \mathrm{ABb}$ & $19,2 \mathrm{~A}$ & 24,1 & 14,4 & $21,2 \mathrm{~A}$ \\
\hline Cascat 953 & $6,9 \mathrm{Ea}$ & $6,6 \mathrm{Ba}$ & $6,2 \mathrm{E}$ & 6,5 & 6,0 & $6,7 \mathrm{D}$ \\
\hline Cascata 797 & $9,8 \mathrm{DEa}$ & $7,6 \mathrm{Ba}$ & $7,6 \mathrm{DE}$ & 8,5 & 6,7 & $8,7 \mathrm{D}$ \\
\hline Cascata 587 & $14,5 \mathrm{CDEa}$ & $7,4 \mathrm{Bb}$ & $9,3 \mathrm{DE}$ & 11,6 & 7,0 & $10,9 \mathrm{CD}$ \\
\hline Conserva 693 & $24,3 \mathrm{ABa}$ & $17,6 \mathrm{Ab}$ & $20,5 \mathrm{~A}$ & 23,8 & 17,3 & $21,0 \mathrm{~A}$ \\
\hline Precocinho & $9,6 \mathrm{DEa}$ & $6,5 \mathrm{Ba}$ & $7,0 \mathrm{DE}$ & 8,3 & 5,7 & $8,0 \mathrm{D}$ \\
\hline Diamante Mejorado & $13,1 \mathrm{CDEa}$ & $6,3 \mathrm{Bb}$ & $12,1 \mathrm{CDE}$ & 12,6 & 11,6 & $9,7 \mathrm{CD}$ \\
\hline Aurora 1 & $19,6 \mathrm{ABCa}$ & $11,6 \mathrm{ABb}$ & $15,7 \mathrm{AB}$ & 19,5 & 11,9 & $15,6 \mathrm{ABC}$ \\
\hline CP 9553 CYN & $9,0 \mathrm{DEa}$ & $5,3 \mathrm{Ba}$ & $7,1 \mathrm{DE}$ & 8,7 & 5,6 & $7,1 \mathrm{D}$ \\
\hline Tropic Beauty & $12,9 \mathrm{CDEa}$ & $7,1 \mathrm{Bb}$ & $20,8 \mathrm{~A}$ & 17,4 & 24,2 & $10,0 \mathrm{CD}$ \\
\hline Douradão & $16,5 \mathrm{BCDa}$ & $7,0 \mathrm{Bb}$ & $10,1 \mathrm{CDE}$ & 12,8 & 7,4 & $11,7 \mathrm{BCD}$ \\
\hline Dourado 2 & $16,7 \mathrm{BCDa}$ & $7,5 \mathrm{Bb}$ & $11,7 \mathrm{CDE}$ & 16,1 & 7,3 & $12,1 \mathrm{BCD}$ \\
\hline Marli & $8,6 \mathrm{DEa}$ & $6,6 \mathrm{Ba}$ & $12,3 \mathrm{BC}$ & 16,3 & 8,3 & $7,6 \mathrm{D}$ \\
\hline Sun Blaze & $17,2 \mathrm{BCDa}$ & $6,7 \mathrm{Bb}$ & $8,0 \mathrm{DE}$ & 8,6 & 7,5 & $11,9 \mathrm{BCD}$ \\
\hline CP 951-C & $16,7 \mathrm{BCDa}$ & $20,0 \mathrm{Aa}$ & 9,9 CDE & 12,6 & 7,1 & $18,4 \mathrm{AB}$ \\
\hline Média & 14,9 & 9,2 & 11,8 & 13,8 & 9,9 & 12,0 \\
\hline
\end{tabular}

Médias seguidas de letras diferentes, maiúsculas na coluna e minúsculas na linha, diferem entre si, pelo teste de Tukey, ao nível de $5 \%$ de probabilidade.

\section{CONCLUSÕES}

1. As aplicações de cianamida hidrogenada e de óleo mineral resultaram na antecipação do início da colheita para todas as cultivares. Também houve concentração no período produtivo, diminuição no ciclo e aumento na produção. A avaliação do ciclo da poda à colheita sob florescimento espontâneo evidenciou que as cultivares mais precoces foram: Precocinho (81,5 dias) e Conserva 693 (87 dias). As mais tardias foram: Turmalina (141,5 dias) e CP 951 C (134,5 dias). Com o uso da cianamida hidrogenada e óleo mineral, o ciclo da poda à colheita permitiu a caracterização das cultivares mais precoces: Precocinho (87,5 dias) e a nectarineira Sun Blaze (95,5 dias). As mais tardias foram: Diamante Mejorado (126,5 dias) e CP 951 C (120 dias).

2. As cultivares Turmalina $\left(20,2 \mathrm{~kg}\right.$ planta $\left.{ }^{-1}\right)$, Conserva 693 (20,75 kg planta-1) e Aurora 1 (15,65 $\mathrm{kg}$ planta $^{-1}$ ) foram as mais produtivas.

\section{REFERÊNCIAS}

ALMEIDA, G.B.V.; DURIGAN, J.F. Relação entre as características químicas e o valor dos pêssegos comercializados pelo sistema veiling frutas Holambra em Paranapanema-SP. Revista Brasileira de Fruticultura, v. 28, p. 218-221, 2006.

BARBOSA, W.; POMMER, C. V.; RIBEIRO, M. D.; VEIGA, R. F. de A.; COSTA, A. A. Distribuição geográfica e diversidade varietal de frutíferas e nozes de clima temperado no Estado de São Paulo. Revista Brasileira de Fruticultura, v. 25, p. 341-344, 2003.

CITADIN, I.; BASSAI, M.H.; DANNER, M.A.; MAZARO, S.M.; GOUVÊA, A. Uso de cianamida hidrogenada e óleo mineral na floração, brotação e produção do pessegueiro 'Chiripá'. Revista Brasileira de Fruticultura, Jaboticabal, v. 28, n. 1, p. 32-35, 2006. 
CUNHA, A.R.; KLOSOWSKI, E.S.; GALVANI, E.; SCOBEDO, J.F.; MARTINS, D. Classificação climática para o município de Botucatu-SP, segundo Köeppen. In: SIMPÓSIO EM ENERGIA NA AGRICULTURA, 1., 1999, Botucatu. Anais. Botucatu: Faculdade de Ciências Agronômicas, Universidade Estadual Paulista, 1999. p.487-491.

EMBRAPA. Centro Nacional de Pesquisa de Solos. Sistema Brasileiro de Classificação de Solos. Rio de Janeiro, 1999. 412 p.

MARODIN, G.A.B.; PEREIRA DIAS, F.; CASTRO, B.; ARGENTA, F. Comportamento de algumas cultivares de pêssegos na depressão central do Rio Grande do Sul In: CONGRESSO BRASILEIRO DE FRUTICULTURA, 20., 2007, Vitória. Anais... Vitória: SBF, 2007. p. 25-30, 2008.

NAVA, G.A.; MARODIN, G.A.B.; PIRES DOS SANTOS, R. Reprodução do pessegueiro:efeito genético, ambiental e de manejo das plantas. Revista Brasileira de Fruticultura, Jaboticabal, v. 31, n.4, p. 1218-1233, 2009.
PEDRO JÚNIOR, M.J.; BARBOSA, W.; ROLIM, G.S.; DE CASTRO, J.L. Época de florescimento e horas de frio para pessegueiros e nectarineiras. Revista Brasileira de Fruticultura. Jaboticabal - SP, v.29, n.3, p.425-430, dezembro de 2007.

PEREIRA, F.M.; MAYER, N.A. Frutificação, características físicas de frutos e produtividade em cultivares e seleções de pessegueiro em Vista Alegre do Alto-SP. Ciência Rural, Santa Maria - RS, v.38, n.6, p. 157-1552, setembro de 2008.

PIRES, E.J.P.; MARTINS, F.P. Técnicas de cultivo. In: POMMER, C.V., ed. Uva: tecnologia de produção, pós-colheita, mercado. Porto Alegre: Cinco Continentes, 2003. p. 351-403. 